\title{
Properties of Crystal States
}

\author{
H. J. F. KNops \\ Instituut voor Theoretische Fysika, Universiteit Nijmegen
}

Received October 24, 1968

\begin{abstract}
It is proved that euclidean invariant states describing crystals are not weakly clustering or equivalently that these states exhibit long range order. Further it is shown that a decomposition of an euclidean invariant state into states all of which are invariant for one specific space group, does not yield states with lattice symmetry.
\end{abstract}

\section{Introduction}

The purpose of this paper is to investigate states describing crystals. Our method will be that of the algebraic approach to statistical mechanics. In this approach one considers states as positive normalised linear functionals on the quasi-local bounded observables which form a $C^{*}$-algebra $\mathfrak{A}$. The euclidean group $E^{3}$ is represented as *automorphisms of $\mathfrak{A}$, its action on $A \in \mathfrak{A}$ is denoted by $\alpha_{g}[A], g \in E^{3}$.

The representation is moreover supposed to be continuous in the sense:

$$
\lim _{g \rightarrow e}\left\|\alpha_{g}[A]-A\right\|=0 .
$$

As is well known this last property is equivalent with the somewhat more physical requirement of weak continuity i.e. continuity of the functions $\phi\left(\alpha_{g}[A]\right)$ for all $A \in \mathfrak{A}$ and all states $\phi[1]$.

In equilibrium statistical mechanics one starts with a state invariant for the euclidean group and one expects that a state describing a crystal is caracterized by the fact that it can be decomposed into states with lower (crystal) symmetry.

One has proposed several methods to perform this decomposition. KASTLER and RoBINSON [2], restricting themselves to translations, made a decomposition into $T_{L}$ extremal invariant states, where $T_{L}$ is the subgroup of translations defined by:

$$
T_{L}=\left\{\boldsymbol{x} \in R^{3} \mid \boldsymbol{x} \cdot \boldsymbol{p}_{n}=0 \quad \bmod 2 \pi, \boldsymbol{p}_{n} \in S_{D}\right\}
$$

$S_{D}$ being the discrete part of the spectrum of the unitary representation of $R^{3}$ corresponding with the state $\phi$ [2].

Their analysis has been extended by RoBinson and Ruelle to the case of the euclidean group [3] (compare also [12]). 
One of the results of this paper is that these decompositions are physically not very relevant. It will be shown that such a decomposition of an euclidean invariant state into states that are invariant for a space group $H \subset E^{3}$, is a decomposition into states that are invariant for the full translation group and delivers therefore no states with real lattice symmetry (Theorem 1). The reason for this failure is in fact that one cannot expect that all the decomposed states are invariant for the same group $H$; in general each will be invariant for a space group conjugate to $H$.

This is precisely what happens in two other, closely related, decompositions. The first one is a decomposition into primary (factor) states [4] the other one in states that are extremal invariant for time translations [5].

The two decompositions are the same for states satisfying the KuboMartin-Schwinger condition [6, 7]; for classical systems however only the latter seems to be of interest [5].

In this paper we will not discuss further the kind of decomposition that one has to make. We simply state that an euclidean invariant state describes a crystal when there exists a decomposition into states $\psi_{\lambda}$ invariant for space groups $H_{\lambda}$.

We show then (Theorem 2) that such a state is not extremal invariant for translations; it is therefore not a clustering state or equivalently it possesses long range order.

This fact is of interest since it is one of the keystones of LANDAU's argument for the non-existence of a critical point for the fluid-solid phase transition.

It looks quite natural that a state describing a crystal has long range order but there seems to be no rigorous proof of this fact [8].

\section{Decomposition into $\boldsymbol{H}$ Invariant States}

Let us start with introducing some more notations.

In the sequel $\mathfrak{A}$ will be a $C^{*}$-algebra with unit element, $\alpha$ a strongly continuous representation of the euclidean group $E^{3}$ as *-automorphisms of $\mathfrak{A}, \alpha^{t}$ the transposed of $\alpha$ in $\mathfrak{A}^{*}$, the dual of $\mathfrak{A} ; \phi$ will denote an euclidean invariant state on $\mathfrak{A}$.

As is well known the G.N.S representation furnishes us with a cyclic representation $\pi$ of $\mathscr{A}$ in $L(\mathscr{H})$ and a unitary representation $U$ of $E^{3}$ such that:

1) $\phi(A)=(\Omega, \pi(A) \Omega), \Omega$ cyclic vector,

2) $\pi\left(\alpha_{g}[A]\right)=U(g) \pi(A) U^{-1}(g)$,

3) $U(g) \Omega=\Omega$. 
The elements of $E^{3}$ will often be explicitly denoted by $(\boldsymbol{a}, R)$ $(\boldsymbol{a}$ : translation, $R$ : rotation) with the multiplication rule: $(\boldsymbol{a}, R) \cdot(\boldsymbol{b}, S)$ $=(\boldsymbol{a}+R(\boldsymbol{b}), R S)$. By SToNE's theorem we know that there exists a projection valued measure $E$ on $R^{3}$ such that:

$$
U(\boldsymbol{a}, 1)=\int e^{-i \boldsymbol{p} \cdot \boldsymbol{a}} d E(\boldsymbol{p}) .
$$

We need the following lemma:

Lemma 1. The discrete spectrum of $U(\boldsymbol{a}, 1)$ contains only $\boldsymbol{p}=0$ or $E(\boldsymbol{p})=0$ when $\boldsymbol{p} \neq 0$.

Proof. Suppose $E(\boldsymbol{p}) \neq 0$ for $\boldsymbol{p}=\boldsymbol{p}_{0} \neq 0$, then there exists a vector $\Psi$ with $\|\Psi\|=1$ such that:

$$
U(\boldsymbol{a}, 1) \Psi=e^{-i \boldsymbol{p}_{0} \cdot \boldsymbol{a}} \Psi \text { for all } \boldsymbol{a} \in R^{3} .
$$

Let $R$ be a rotation then

hence

$$
U^{-1}(0, R) U(\boldsymbol{a}, 1) U(0, R)=U\left(R^{-1}(\boldsymbol{a}), 1\right)
$$

or

$$
U^{-1}(0, R) U(\boldsymbol{a}, 1) U(0, R) \Psi=e^{-i \boldsymbol{p}_{0} \cdot R^{-1}(\boldsymbol{a})} \Psi
$$

$$
U(\boldsymbol{a}, 1) U(0, R) \Psi=e^{-i \boldsymbol{p}_{0} \cdot R^{-1}(\boldsymbol{a})} U(0, R) \Psi .
$$

Because $\boldsymbol{p}_{0} \neq 0$ there exists rotations arbitrary close to 1 such that:

$$
e^{-i \boldsymbol{p}_{0} \cdot \boldsymbol{a}} \neq e^{-i \boldsymbol{p}_{0} \cdot R^{-1}(\boldsymbol{a})}
$$

and for such rotations $\Psi$ and $U(0, R) \Psi$ are eigenvectors of $U(\boldsymbol{a}, 1)$ with different eigenvalues, hence $(U(0, R) \Psi, \Psi)=0$.

This implies then

$$
\|U(0, R) \Psi-\Psi\|^{2}=2
$$

which is in contradiction with the continuity of the representation $U$. We remark in passing that with somewhat more elaborate tools it is possible to derive a stronger result, namely:

$$
\lim _{a \rightarrow \infty}(\Psi, U(\boldsymbol{a}, 1) \Phi)=\left(\Psi, P_{0} \Phi\right)
$$

$\Psi$ and $\Phi$ vectors of $\mathscr{H}$ and $P_{0}$ projection on the subspace of $\mathscr{H}$ formed by vectors invariant under $U\left(R^{3}, 1\right)[3]$.

The lemma above will however be sufficient for our purposes.

We now prove the following theorem.

Theorem 1. Let $\phi$ be a state invariant for $E^{3}$ and let there be given a decomposition of $\phi$ :

$$
\phi=\int_{X} \psi_{x} d \mu(x)
$$

where $X$ is a compact space and $\mu$ is a Radon measure on $X$. Further it is supposed that the map $x \rightarrow \psi_{x}(A)$ is continuous for fixed $A \in \mathfrak{Z}$ and that $\psi_{x}$ is invariant for a subgroup $H$ of $E^{3}$ that contains at least three noncoplanar translations $\boldsymbol{a}_{1}, \boldsymbol{a}_{2}, \boldsymbol{a}_{3}$. Then $\psi_{x}$ is invariant for all translations when $x \in \operatorname{supp}(\mu)$. 
Notice that the decompositions in [2] and [3] fulfill indeed the conditions above, $X$ being $E / H$ and $\psi_{x}=\alpha_{g}^{t} \psi, g$ contained in the equivalence class $x$.

Proof. We take $\phi_{D}=\int_{D} \psi_{x} d \mu(x)$ for $D$ a measurable set of $X$. Then clearly $\phi_{D} \leqq \phi$, hence there is a vector $\Omega_{D} \in \mathscr{H}$ such that $\phi_{D}(A)$ $=\left(\Omega_{D}, \pi(A) \Omega\right)([9], 2.5 .1)$. Since $\psi_{x}$ is invariant for $H, \phi_{D}$ is invariant for $H$ and therefore

$$
U(g) \Omega_{D}=\Omega_{D} \text { for } \quad g \in H .
$$

In particular we have

$$
U\left(\boldsymbol{a}_{i}, 1\right) \Omega_{D}=\Omega_{D} \quad(i=1,2,3) .
$$

Remembering now the spectral resolution of $U$

one has:

$$
U\left(\boldsymbol{a}_{i}, 1\right)=\int e^{-i \boldsymbol{p} \cdot \boldsymbol{a}_{i}} d E(\boldsymbol{p})
$$

$$
E\left(0 \cup \boldsymbol{p}_{1} \cup \boldsymbol{p}_{2} \cup \cdots\right) \Omega_{D}=\Omega_{D} .
$$

Where the $\boldsymbol{p}_{j}$ are the points of the dual lattice i.e.

$$
\boldsymbol{p}_{j} \cdot \boldsymbol{a}_{i}=0 \bmod 2 \pi \quad(i=1,2,3) .
$$

The dual lattice is discrete, since $\boldsymbol{a}_{1}, \boldsymbol{a}_{2}, \boldsymbol{a}_{3}$ are non-coplanar. When we use now the $\sigma$-additivity of the measure $E$ and Lemma 1 we deduce:

$$
E(0) \Omega_{D}=\Omega_{D} \text { or } \Omega_{D} \quad \text { is invariant for } U(\boldsymbol{a}, 1), \quad \boldsymbol{a} \in R^{3} .
$$

Hence $\phi_{D}\left(\alpha_{(\boldsymbol{a}, 1)}[A]-A\right)=0$ for all $\boldsymbol{a} \in R^{3}$ or explicitly:

$$
\int_{D}\left\{\psi_{x}\left(\alpha_{(\boldsymbol{a}, 1)}[A]\right)-\psi_{x}(A)\right\} d \mu(x)=0 .
$$

This can only be true for every measurable set $D$ when $\psi_{x}\left(\alpha_{(\boldsymbol{a}, 1)}[A]\right)$ $=\psi_{x}(A)$ for almost all $x \in X$ (e.g. [10], $25 \mathrm{E}$ ).

Since we assumed the map $x \rightarrow \psi_{x}(A)$ to be continuous the equality holds in fact for all $x \in \operatorname{supp}(\mu)$ (e.g. [11], III, 3).

This ends the proof of the theorem.

We notice that one can consider this theorem as a generalization of a theorem of Robinson and Ruelce ([3], Th. 2) which states that an euclidean invariant state that is furthermore strongly clustering cannot be decomposed into states invariant for $H$ in a non trivial way.

\section{Long Range Order}

We start the proof of the presence of long range order for states describing crystals with a definition and a lemma:

Definition. Let $\psi$ be a state on $\mathfrak{A}$ then $\eta_{R} \psi$ and $\eta \psi$ are defined respectively by:

$$
\eta_{R} \psi(A)=\frac{1}{4 / 3 \pi R^{3}} \int_{B(R)} \alpha_{(\boldsymbol{a}, 1)}^{t} \psi(A) d \boldsymbol{a} ; \quad B(R)=\left\{\boldsymbol{a} \in R^{3}|| \boldsymbol{a} \mid<R\right\}
$$

and $\eta \psi(A)=\lim _{R \rightarrow \infty} \eta_{R} \psi(A)$ whenever this limit exists for all $A \in \mathfrak{A}$. 
It is clear that whenever $\eta \psi$ exists it is a state invariant for all translations.

Lemma 2. Suppose $\psi$ is invariant for a space group $H$ and let the lattice be generated by $\boldsymbol{a}_{1}, \boldsymbol{a}_{2}, \boldsymbol{a}_{3}$. Then $\eta \psi$ exists and when $\Delta$ is the elementary cell and $v(\Delta)$ its volume then:

$$
\eta \psi(A)=\frac{1}{v(\Delta)} \int_{\Delta} \alpha_{(\boldsymbol{a}, 1)}^{t} \psi(A) d \boldsymbol{a} .
$$

When furthermore $\psi$ is not invariant for the full translation yroup then $\eta \psi$ is not euclidean invariant.

Proof. The first assertion is easily seen to be true because one can, by making use of the invariance of $\psi$ for $\boldsymbol{a}_{1}, \boldsymbol{a}_{2}, \boldsymbol{a}_{3}$, reduce the integration over cells inside $B(R)$ to an integration over the elementary cell situated at the origin. There remains then only an integration over cells at the surface of $B(R)$ that are only partly in $B(R)$ but the total volume of these cells is of the order of the surface of $B(R)$; therefore this gives a contribution that vanishes when we divide by $R^{3}$ and take the limit $R \rightarrow \infty$ (notice that $\left|\alpha_{\boldsymbol{a}}^{t} \psi(A)\right|$ is bounded by $\|A\|$ ). The second assertion is a direct consequence of Theorem 1 since the integration over $\Delta$ satisfies all conditions of this theorem.

We have gathered now all preliminairies to prove the following theorem.

Theorem 2. Let $\phi$ be a state on $\mathfrak{A}$ invariant for the euclidean group $E^{3}$. Suppose $\phi$ can be decomposed into crystal-states i.e.

$$
\phi(A)=\int_{\Lambda} \Psi_{\lambda}(A) d \mu(\lambda)
$$

with $\Lambda$ a measure space $(\mu(\Lambda)=1$ take $A=1)$, Suppose further that for almost all $\lambda \in \Lambda$ there is a space-group $H_{\lambda} \subset E$ for which the state $\psi_{\lambda}$ is invariant and that the $\psi_{\lambda}$ are not invariant for the full translation group. Then $\phi$ is not extremal invariant for translations.

Proof. We prove this by explicitly constructing a decomposition into translation invariant states.

The invariance of $\phi$ for $E^{3}$ gives:

$$
\phi(A)=\eta_{R} \phi(A)=\frac{1}{4 / 3 \pi R^{3}} \int_{B(R)} d \boldsymbol{a} \int_{A} \alpha_{(\boldsymbol{a}, 1)}^{t} \psi_{\lambda}(A) d \mu\left(\lambda_{\nu}\right)
$$

When $A$ is fixed $\alpha_{(\boldsymbol{a}, 1)}^{t} \psi_{\lambda}(A)$ is a function from $B(R) \times A$ to $C$. This function is for fixed $\boldsymbol{a}$ measurable in $\lambda$ (otherwise the decomposition would not make sense).

Furthermore the fact that the euclidean group is continuously represented implies that the function is uniformly continuous in $\boldsymbol{a}$ for all 
$\lambda$. This is sufficient to guarantee that the function is measurable on the product space because one can approximate it arbitrarily close by functions that are piecewise constant in $\boldsymbol{a}$ and therefore measurable.

Since the function is moreover bounded (by $\|A\|$ ) and its support has finite measure it is integrable on $B(R) \times A$. We may apply then Fubini's theorem (e.g. [10], $36 \mathrm{C}$ ) and interchange the order of integration:

$$
\phi(A)=\int_{\Lambda} d \mu(\lambda) \frac{1}{4 / 3 \pi R^{3}} \int_{B(R)} \alpha_{(\boldsymbol{a}, 1)}^{t} \psi_{\lambda}(A) d \boldsymbol{a}=\int_{\Lambda} d \mu(\lambda) \eta_{\lambda} \psi_{\lambda}(A) .
$$

We now take the limit $R \rightarrow \infty$, this exists for all crystal states (Lemma 2) hence for almost all $\lambda: \lim _{R \rightarrow \infty} \eta_{R} \psi_{\lambda}(A)=\eta \psi_{\lambda}(A)$.

Moreover all the functions $\eta_{R} \psi_{\lambda}(A)$ are bounded from above by $\|A\|$ hence we may use Lebesgue's bounded convergence theorem (e.g. [10], $26 \mathrm{D}$ ) and interchange the limit $R \rightarrow \infty$ and the integration over $A$ resulting in:

$$
\phi(A)=\int_{1} d \mu(\lambda) \eta \psi_{\lambda}(A)
$$

Now $\eta \psi_{\lambda}$ is invariant for translations but still not invariant for the euclidean group (Lemma 2). Therefore $\eta \psi_{\lambda}$ cannot be a multiple of $\phi$ and we achieved a non-trivial decomposition of $\phi$ into translation invariant states.

The presence of long range order in $\phi$ or equivalently the absence of cluster properties follows from this fact by standard arguments (e.g. $[1,2])$.

Acknowledgements. The author is indebted to Prof. E. J. Verboven for suggesting the present investigation and for critical reading the manuscript. Further he would like to express his gratitude to Prof. G. G. Emcr for many enlightening discussions during the initial stage of this work and to Dr. W. WILs for interesting conversations on the mathematical aspects of the problem.

\section{References}

1. Doplicher, S., D. Kastler, and D. Robinson: Commun. Math. Phys. 3, 1 (1966).

2. Kastler, D., and D. Robinson: Commun. Math. Phys. 3, 151 (1966).

3. Robinsox, D., and D. Ruelle: Ann. Inst. H. Poincaré VI. 4, 299 (1967).

4. Kastler, D., R. HaAg, and L. Michel: Central decompositions of ergodic states. Preprint, Marseille 1967.

5. Emch, G., H. KNops, and E. Verboven: Proceedings of the international conference on statistical mechanics. Kyoto: The Physical Society of Japan 1968.

6. WINNINK, M.: An application of $C^{*}$-algebras to quantum statistical mechanics of systems in equilibrium. Thesis, Groningen 1968. 
7. Araki, H., and H. Mryata: On KMS boundary condition. Preprint. Kyoto University 1968.

8. UhlenbecK, G.: Fundamental problems in statistical mechanics II, p. 17. Amsterdam: North Holland 1968.

9. Drxmier, J.: Les $C^{*}$-algèbres et leurs répresentations. Paris: Gauthier-Villars 1964.

10. Halmos: Measure theory. Princeton: Van Nostrand Comp. 1956.

11. Bourbaki, N.: Livre VI Intégration. Paris: Hermann 1952.

12. Doplicher, S., and D. Kastrler: Commun. Math. Phys. 7, 1 (1968).

Drs. H. J. F. KNops

Instituut voor Theoretische Fysika

Universiteit Nijmegen

Nijmegen, Holland 